\title{
LOWER BOUNDS FOR THE UNKNOTTING NUMBERS OF CERTAIN TORUS KNOTS
}

\author{
MAKOTO YAMAMOTO
}

\begin{abstract}
In this paper we shall show that the unknotting numbers of the $(l, 2 k l \pm 1)$-torus knots are at least $\left(k\left(l^{2}-1\right)-2\right) / 2$ for $l$ odd and $\left(k l^{2}-2\right) / 2$ for $l$ even, where $l$ is an integer greater than one and $k$ is a positive integer.
\end{abstract}

1. Introduction. Unless otherwise stated all manifolds and maps are smooth and all knots and links are in $S^{3}$.

The unknotting number of a knot is the minimum number of crossings which must be changed to make the knot trivial. Let $l$ and $m$ be integers. The $(l, m)$-torus link is the link which lies on an unknotted torus and sweeps around it $l$ times in the longitude and $m$ times in the meridian. When $l$ and $m$ are relatively prime, it is a knot and called the $(l, m)$-torus knot. Milnor [3] conjectured that the unknotting number of a torus knot is equal to the genus of it. It is well known that the genus of the $(l, m)$-torus knot is equal to $(l-1)(m-1) / 2$. It is not hard to see that the unknotting number of a torus knot is at most the genus of it. In this paper we shall show the following.

THEOREM A. Let $l$ be an integer greater than one and $k$ a positive integer. Then the unknotting numbers of the $(l, 2 k l \pm 1)$-torus knots are at least $\left(k\left(l^{2}-1\right)-2\right) / 2$ if $l$ is odd, and $\left(k l^{2}-2\right) / 2$ if $l$ is even.

Murasugi [4] showed that the unknotting number of the $(2, m)$-torus knot is equal to the genus of it. Weintraub [8] showed that the unknotting number of the $(m-1, m)$-torus knot is at least $\left(m^{2}-5\right) / 4$ if $m$ is odd, and $\left(m^{2}-4\right) / 4$ if $m$ is even.

The author wishes to thank Professors Mitsuyoshi Kato and Hiroshi Noguchi for helpful suggestions.

2. Preliminaries. The following is a theorem of Rohlin [5], Hsiang-Szczarba [9], Thomas-Wood [6] and Weintraub [7].

THEOREM 1. Let $N$ be an oriented, connected, simply connected, closed 4manifold. Let $M$ be an oriented, connected, closed surface embedded in $N$. Suppose that $M$ represents a 2-homology class $[M]$ of $H_{2}(N ; Z)$ and that $[M]$ is divisible by a positive integer $d$ in the free abelian group $H_{2}(N ; Z)$. Let $g_{M}$ be the genus of $M$. Then

$$
2 g_{M} \geq \frac{[M]^{2}}{d^{2}} \frac{d^{2}-1}{2}-\operatorname{rank} H_{2}(N ; Z)-\operatorname{signature} N
$$

for $d$ odd, and similarly for $d$ even with $d^{2} / 2$ instead of $\left(d^{2}-1\right) / 2$, where $[M]^{2}$ is the self-intersection number of $M$.

Received by the editors August 26, 1981.

1980 Mathematics Subject Classification. Primary 57M25.

Key words and phrases. Torus knot, torus link, unknotting number of a knot, genus of a knot. 


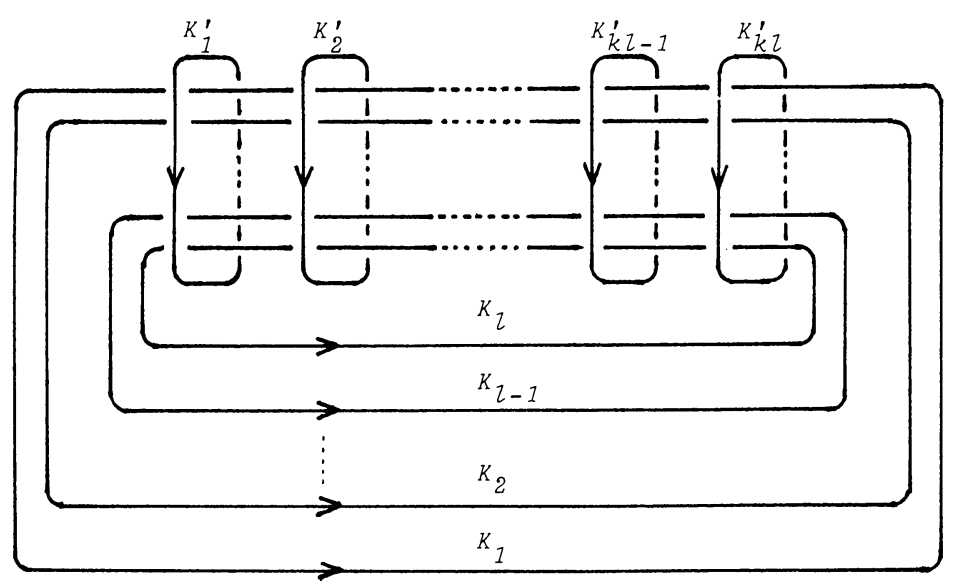

Figure 1

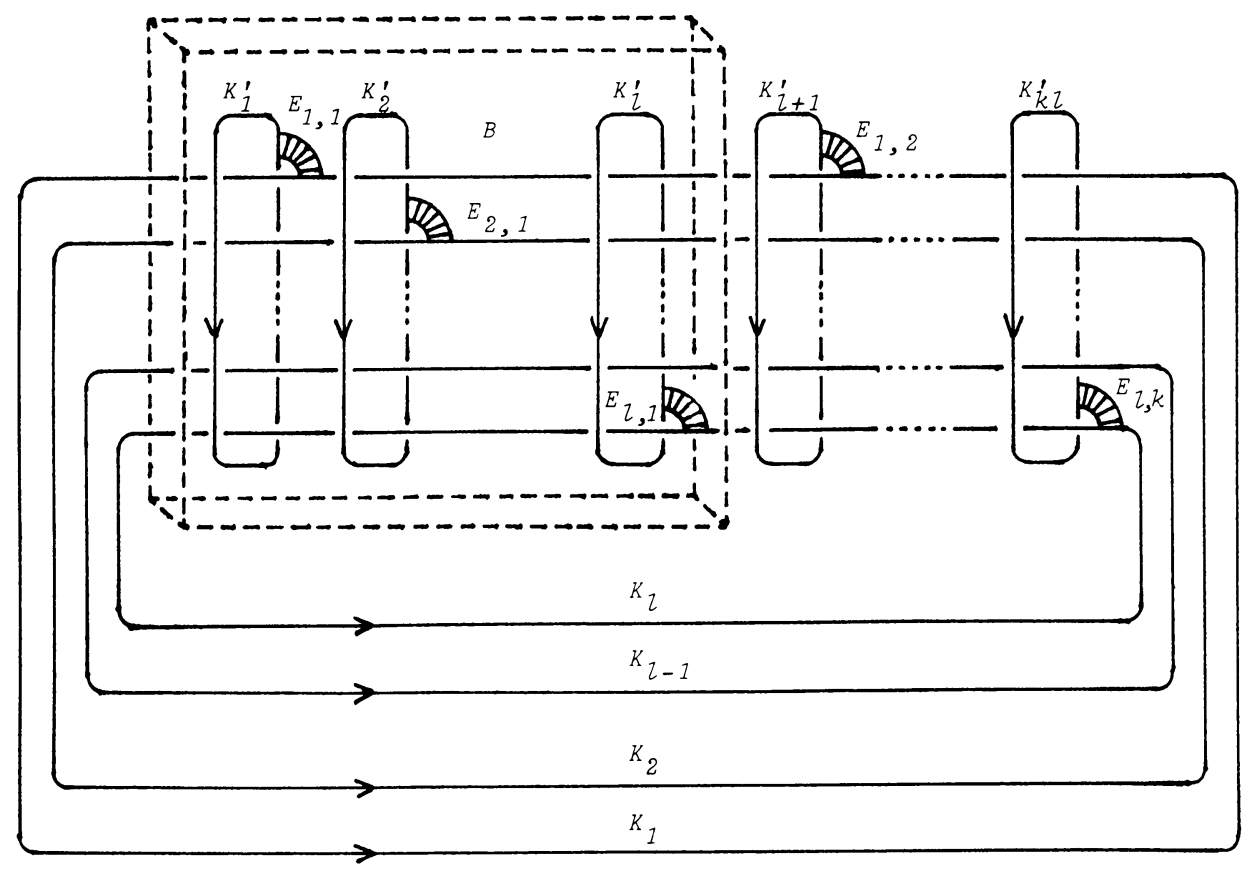

FIGURE 2

The following theorem originally due to Boardman [1] and Weintraub [8].

THEOREM 2. Let $N$ be an oriented 4-manifold, and let $N_{0}=N-\operatorname{Int} B^{4}$, where $B^{4}$ is an embedded 4-ball. Suppose $\alpha \in H_{2}\left(N_{0}, \partial N_{0} ; Z\right)$ is represented by an embed$\operatorname{ding} \Phi:\left(B^{2}, S^{1}\right) \rightarrow\left(N_{0}, \partial N_{0}\right)$ and let $K$ denote the knot given by $\Phi \mid S^{1}: S^{1} \rightarrow \partial N_{0}=$ $S^{3}$. If $u$ is the unknotting number of $K$, then $\alpha$ is represented by an embedded 2sphere in $N \#(u W)$, where $W=C P^{2} \#\left(-C P^{2}\right)$.

The proof is given by using $W$ instead of $C P^{2}$ and $-C P^{2}$ of Theorem 7 of [8]. 


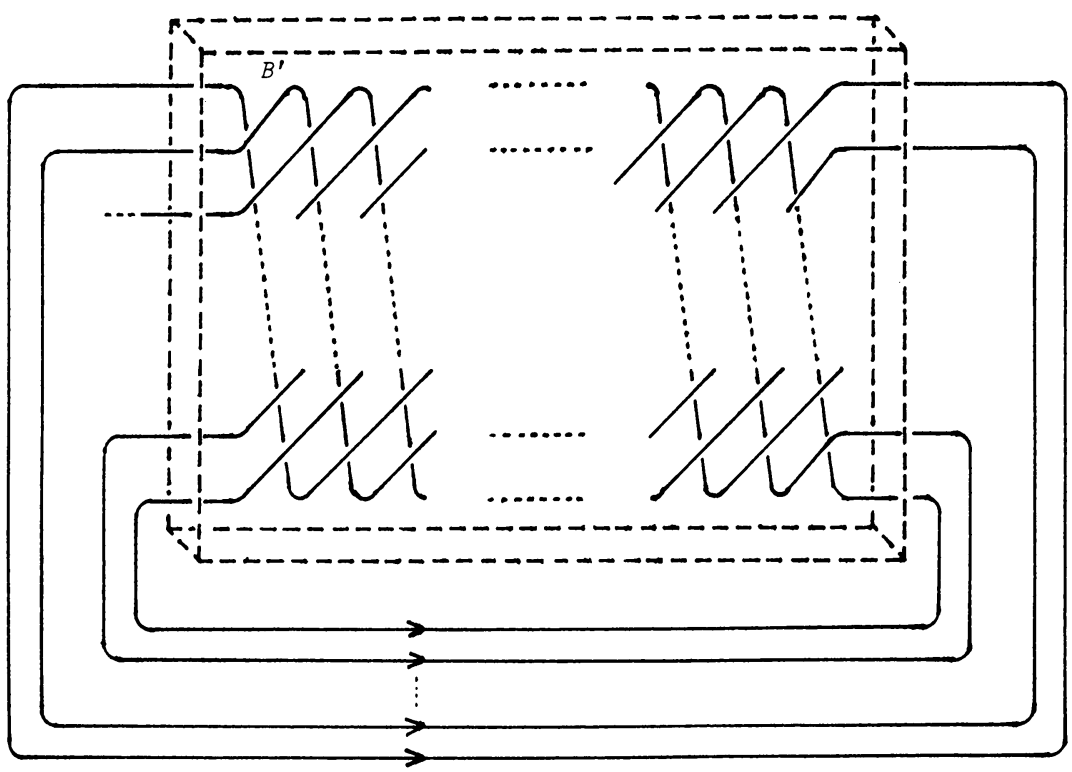

Figure 3

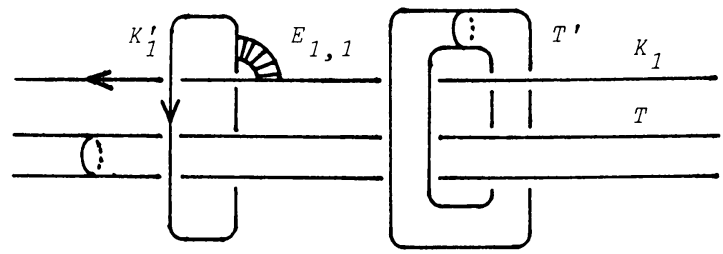

Figure 4

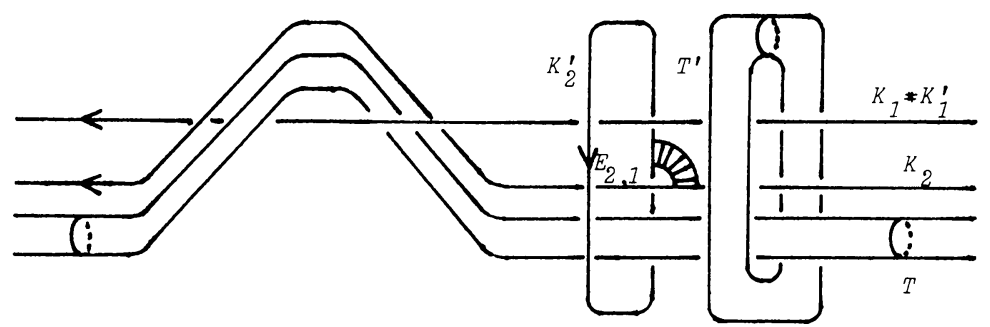

FIGURE 5

3. Proof of Theorem A. Let $\alpha$ and $\beta$ be the generators of

$$
H_{2}\left(S^{2} \times S^{2}-\operatorname{Int} B^{4}, \partial\left(S^{2} \times S^{2}-\operatorname{Int} B^{4}\right) ; Z\right),
$$

and let $l$ and $m$ be positive integers. Then $l \alpha+m \beta$ can be represented by an embedded disk, say $D_{l, m}$. Let $K_{l, m}$ be the knot which is the boundary of $D_{l, m}$. 


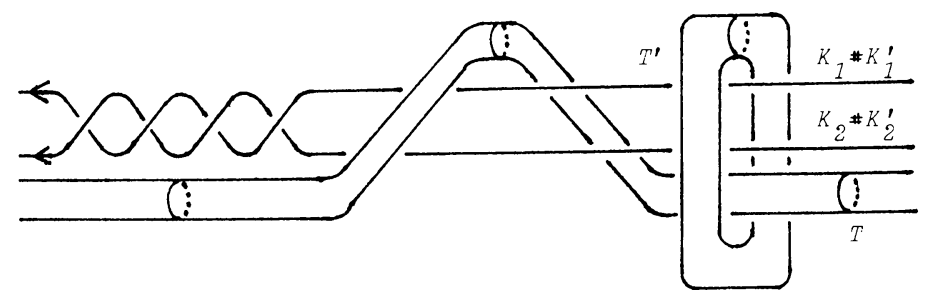

FIGURE 6

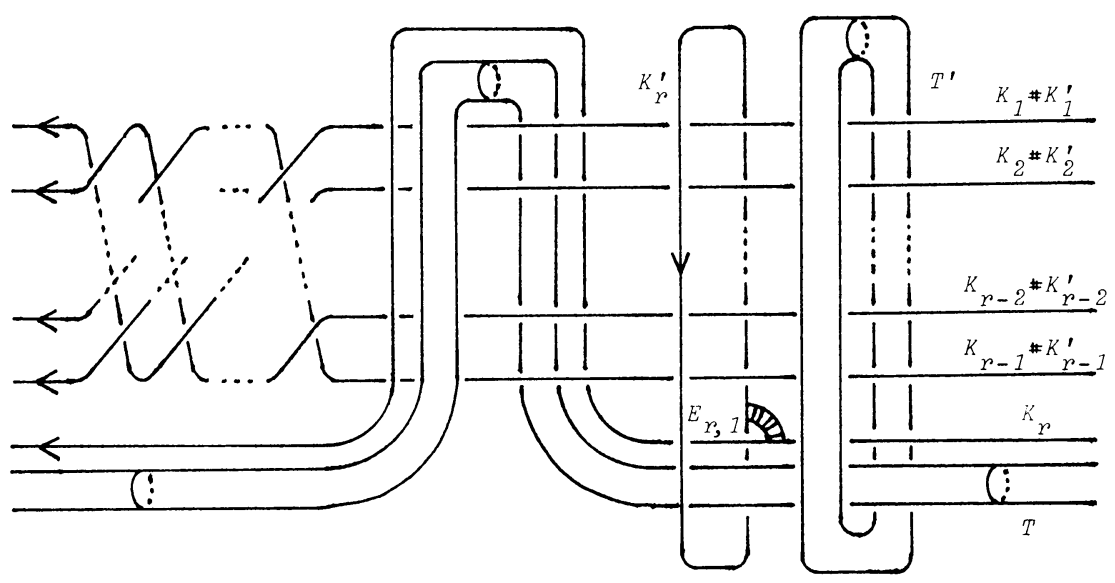

FIGURE 7

LEMMA. Let $u$ be the unknotting number of $K_{l, m}$. Suppose that $l$ and $m$ are divisible by a positive integer $d$. Then

$$
u \geq \begin{cases}\frac{\operatorname{lm}\left(d^{2}-1\right)}{2 d^{2}}-1 & \text { if dis odd } \\ \frac{\operatorname{lm}}{2}-1 & \text { if dis even } .\end{cases}
$$

Proof. By Theorem 2, $l \alpha+m \beta$ is represented by an embedded 2-sphere in $\left(S^{2} \times S^{2}\right) \#(u W)$. Then, by Theorem 1 ,

and

$$
0 \geq \frac{2 l m}{d^{2}} \frac{d^{2}-1}{2}-2(1+u) \text { if } d \text { is odd }
$$

$$
0 \geq \frac{2 l m}{d^{2}} \frac{d^{2}}{2}-2(1+u) \text { if } d \text { is even, }
$$

and elementary algebra yields the lemma.

Let $l$ be an integer greater than one, and let $k$ be a positive integer. We show that the class

$$
l \alpha+k l \beta \in H_{2}\left(S^{2} \times S^{2}-\operatorname{Int} B^{4}, \partial\left(S^{2} \times S^{2}-\operatorname{Int} B^{4}\right) ; Z\right)
$$

can be represented by an embedded disk with boundary the $(l, 2 k l \pm 1)$-torus knot. The class $l \alpha+k l \beta$ can be represented by $l+k l$ embedded disks with boundary the link $L$ of $l+k l$ components illustrated in Figure 1 . Let $K_{1}, \ldots, K_{l}, K_{1}^{\prime}, \ldots, K_{k l}^{\prime}$ 


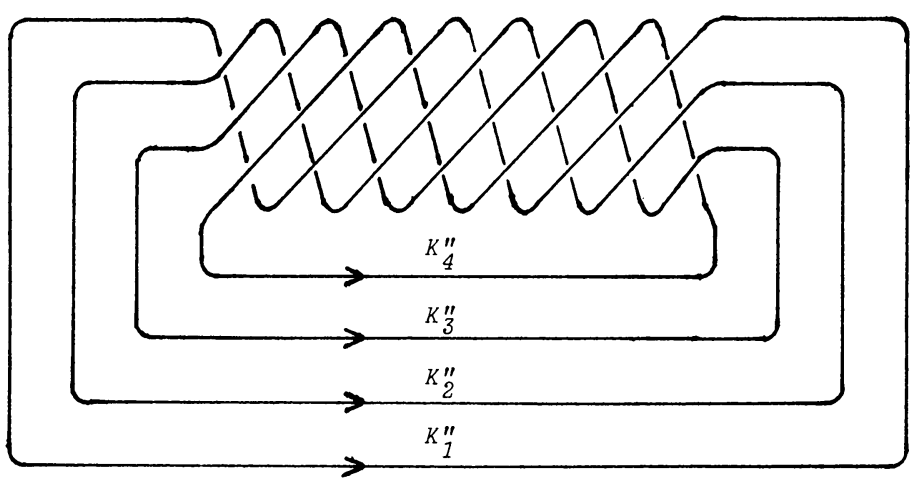

The (4,8)-torus link

Figure 8

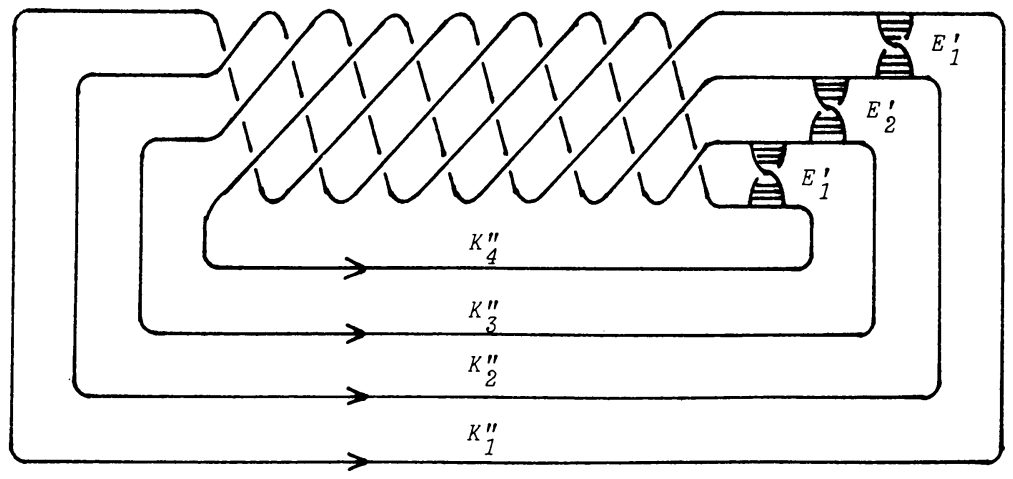

FiguRE 9

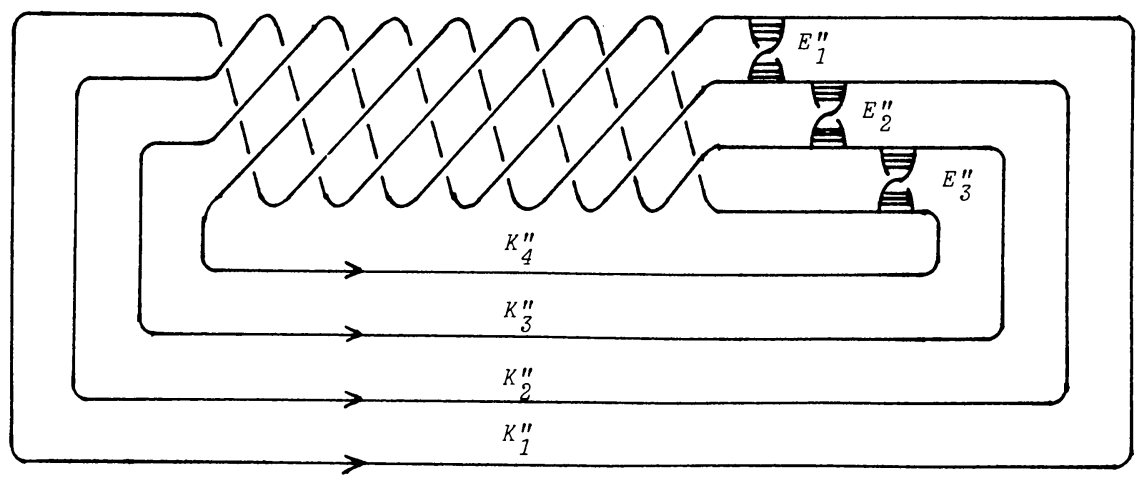

FIGURE 10

be as in Figure 1. Then $l \alpha+k l \beta$ can be represented by the disk obtained by connecting $K_{1}, \ldots, K_{l}, K_{1}^{\prime}, \ldots, K_{k l}^{\prime}$ by $l+k l-1$ strips in $\partial\left(S^{2} \times S^{2}-\operatorname{Int} B^{4}\right)=S^{3}$ (see Kervaire-Milnor [2]). We connect $K_{i}$ and $K_{(j-1) l+i}^{\prime}$ by the strip $E_{i, j}$ as in Figure 2 for $i=1, \ldots, l$ and $j=1, \ldots, k$, thereby obtaining a collection of $l$ disks representing the class $l \alpha+k l \beta$, whose boundaries form a link of $l$ components. 
Let $L^{\prime}$ be the link which consists of the boundaries of the disks obtained by the above construction. We show that the link $L^{\prime}$ is the $(l, 2 k l)$-torus link as follows; it is sufficient to show that there is an isotopy of $S^{3}$ which deforms the part of $L^{\prime}$ contained in the 3-ball $B$ in Figure 2 into the part of the $(l, 2 l)$-torus link contained in the 3-ball $B^{\prime}$ in Figure 3 and which is relative to the complement of $B$. In Figures 4, 5, 6 and 7 we illustrate only the parts contained in the 3-balls, and all isotopies are relative to the complements of the 3-balls. We may consider that the components $K_{2}, \ldots, K_{l}\left(K_{2}^{\prime}, \ldots, K_{l}^{\prime}\right)$ of $L^{\prime}$ are contained in a narrow tube $T$ (resp. $\left.T^{\prime}\right)$ as in Figure 4. We connect $K_{1}$ and $K_{1}^{\prime}$ by the strip $E_{1,1}$. Then we have the link which is isotopic to the link illustrated in Figure 5. We take $K_{2}\left(K_{2}^{\prime}\right)$ out of $T$ (resp. $T^{\prime}$ ) and connect $K_{2}$ and $K_{2}^{\prime}$ by the strip $E_{2,1}$. Then we have the link which is isotopic to the link as in Figure 6, where the components $K_{1} \# K_{1}^{\prime}$ and $K_{2} \# K_{2}^{\prime}$ form the $(2,4)$-torus link. We suppose that we have the link as in Figure 7 after connecting $K_{i}$ and $K_{i}^{\prime}$ by the strip $E_{i, 1}$ for $i=1, \ldots, r-1 \quad(2 \leq r \leq l-1)$ as above and that the components $K_{1} \# K_{1}^{\prime}, K_{2} \# K_{2}^{\prime}, \ldots, K_{r-1} \# K_{r-1}^{\prime}$ form the $(r-1,2(r-1))$-torus link. We connect $K_{r}$ and $K_{r}^{\prime}$ by the strip $E_{r, 1}$ as in Figure 7. Then we have the link which is isotopic to the similar link as in Figure 7 with $K_{r-1} \# K_{r-1}^{\prime}, K_{r} \# K_{r}^{\prime}, K_{r+1}$ and $K_{r+1}^{\prime}$ instead of $K_{r-2} \# K_{r-2}^{\prime}, K_{r-1} \# K_{r-1}^{\prime}$, $K_{r}$ and $K_{r}^{\prime}$. Then we have inductively the link $\left(K_{1} \# K_{1}^{\prime}\right) \cup \cdots \cup\left(K_{l} \# K_{l}^{\prime}\right)$ which is isotopic to the $(l, 2 l)$-torus link by the composite of the above isotopies as required. Therefore we have shown that the link $L^{\prime}$ is the $(l, 2 k l)$-torus link.

The $(l, 2 k l)$-torus link is represented as in Figure 8 . Let $K_{i}^{\prime \prime}$ be the component $K_{i} \# K_{i}^{\prime} \# K_{l+i}^{\prime} \# \ldots \# K_{(k-1) l+i}^{\prime}$ of $L^{\prime}$. We connect $K_{i}^{\prime \prime}$ and $K_{i+1}^{\prime \prime}(i=1, \ldots, l-1)$ by the strip $E_{i}^{\prime}$ as in Figure 9 . Then we have the $(l, 2 k l-1)$-torus knot. When we connect $K_{i}^{\prime \prime}$ and $K_{i+1}^{\prime \prime}(i=1, \ldots, l-1)$ by the strip $E_{i}^{\prime \prime}$ as in Figure 10, we have the $(l, 2 k l+1)$-torus knot. By Lemma, we obtain Theorem A.

\section{REFERENCES}

1. J. Boardman, Some embeddings of 2-spheres in 4-manifolds, Proc. Cambridge Philos. Soc. 60 (1964), 354-356.

2. M. Kervaire and J. Milnor, On 2-spheres in 4-manifolds, Proc. Nat. Acad. Sci. U.S.A. 47 (1961), 1651-1657.

3. J. Milnor, Singular points of complex hypersurfaces, Ann. of Math. Studies, No. 61, Princeton Univ. Press, Princeton, N.J., 1968.

4. K. Murasugi, On a certain numerical invariant of link types, Trans. Amer. Math. Soc. 117 (1965), $387-422$.

5. V. A. Rohlin, Two-dimensional submanifolds of four dimensional manifolds, Functional Anal. Appl. 5 (1971), 39-48.

6. P. E. Thomas and J. Wood, On manifolds representing homology classes in codimension 2 , Invent. Math. 25 (1974), 63-89.

7. S. H. Weintraub, $Z_{p}$-actions and rank of $H_{2}\left(N^{2 n}\right)$, J. London Math. Soc. (2) 13 (1976), 567572 .

8. - Inefficiently embedded surfaces in 4-manifolds, Algebraic Topology Aarhus 1978 (J. L. Dupont and I. H. Madsen, eds.), Lecture Notes in Math., vol. 763, Springer-Verlag, Berlin and New York, 1979.

9. W. C. Hsiang and R. H. Szczarba, On embedding surfaces in four-manifolds, Algebraic Topology, Proc. Sympos. Pure Math., Vol. 22, Amer. Math. Soc., Providence, R.I., 1971.

DePaRtMENT OF MATHEMATICS, SCHOOL OF SCIENCE AND ENGINEERING, WASEDA UNIVERSITY, SHINJUKU, TOKYO, 160, JAPAN 